\title{
Kinematic characteristics of the car movement from the top to the calculation point of the marshalling hump
}

\author{
Shukhrat Saidivaliev", Ramazon Bozorov, and Elbek Shermatov \\ Tashkent State Transport University, Tashkent, Uzbekistan
}

\begin{abstract}
Introduce analytical acceleration formulas that are derived from the classic d'Alembert principle of theoretical mechanics for high-speed sections and sections of retarder positions; show the possibility of determining the instantaneous car speeds in each section of the marshalling hump according to the formulas of elementary physics both for high-speed sections and for sections of retarder positions; provide formulas for determining the time of movement of a car with uniformly accelerated and/or uniformly retarded motion of the car on the inclined part of the hump, as well as in areas of retarder positions. Research methods: The classic d'Alembert principle of theoretical mechanics is widely used in the paper. Main results: For the first time, the results of constructing a graphical dependence of the estimated height of the marshalling hump over the entire length of its profile are presented in the form of a decrease in the profile height of each section of the inclined part in proportion to the slope of the track. The results of constructing graphical dependences on changes in the speed and time of movement of a car along the entire length of the inclined part of the marshalling hump are fundamentally different from the existing methodology, where, for example, curves of medium (rather than instantaneous) speeds of a car are built. The proposed new methodology for calculating the kinematic characteristics of the car movement along the entire length of the hump allows an analysis of the mode of shunting car at the marshalling humps.
\end{abstract}

\section{Introduction}

Railway vehicles are transport systems designed for safe and smooth movement on individual tracks. They differ significantly from automobiles and other transport systems. They operate on tracks consisting of straight and curved sections and use propulsion and brake system without active steering means, such as wheel steering systems or rudders. At the same time, railway vehicles must satisfy conflicting demands: straight-line stability on straight-line tracks (running stability) and turning on curved tracks. In addition, they need to maintain high ride quality and be immune to vibrations when passing through irregular sections of track or switches. In other words, it is very important that railway vehicles have good running stability and turning characteristics while ensuring good ride quality [1].

\footnotetext{
* Corresponding author: shuxratxoja@mail.ru
} 
The railway industry is essentially conservative. Only in recent years have modern scientific methods of analysis been applied to the problem of rail vehicle dynamics. Its complexity is extreme, but the need for increased velocity and greater carrying capacity, both of which create new problems in terms of wear and stability, forces railway operators and equipment suppliers to solve these problems more systematically and fundamentally [2$4]$.

[5] studies the vehicle's dynamic behaviour and track over a long and inclined section of a high-velocity railway under braking conditions. A model of the dynamic interaction of the vehicle and the track was built based on two longitudinal models of that interaction. In the model, the vehicle is considered a multi-rigid system with 21 degrees of freedom, consisting of an automobile body, two railroad trucks and four wheels; using the finite element method, the rail track is modeled as an Euler beam; the method of "circular path" to reduce the degree of freedom of the model for modeling a long journey; Two models of the longitudinal interaction of a wheel and a rail are considered: the Polach creep theory (suitable for modeling high creep as a result of strong braking) [14] and the longitudinal theory of hard contact. The dynamic characteristics of substructures during vehicle braking, calculated using models based on the Polach creep model and longitudinal contact models, show a small difference, but the Polach creep model can fully take into account wheel movement, and greater wheel-rail creep during braking and can accurately analyze damage to the wheel-rail interface.

Analysis of dynamic interaction between the wheel and the rail under various conditions shows that a large braking torque will cause some or all wheels to slide, damaging the wheel-rail contact. This will increase the braking distance and time and extend the sliding time of the locked wheels, increasing the risk of damage to the wheel from rail contact. Braking torque should be kept below a reasonable value so the braking distance and braking time can be as short as possible without causing the wheel to slip along the track. According to the calculations performed in this study, a reasonable braking torque under dry conditions and wet conditions should be 7 and $4 \mathrm{kN} \cdot \mathrm{m}$, respectively.

Several works have investigated the dynamics of a braking railway car in retarder positions $[6,7]$. An important task is regulating velocities in railcar braking areas (BA) and retarder positions (RP). High capacity hump yards are equipped with various kinds of car retarders, which are the primary means for regulating the velocity of rolling stock [8]. Meanwhile, two types of braking are required - interval and sight (targeted). Interval braking provides the necessary intervals between cars for their safe passage through turnouts and braking devices in the classification bowl. Sight (targeted) braking allows the car's velocity to be adjusted depending on the distance that it must go in the hump yard. [9] describes a variety of factors (propulsion properties of railcars, their gravity, range, curves and straightaways in the study path along the profile of the yard, weather conditions, and the human factor) that affect the difficulty of railcar braking in hump yards. It also describes the purpose and importance of applying for each braking position (first, second, third - RP1, RP2, RP3).

The transport task of determining the travel time and braking distance of a railcar in a brake position area was considered intractable [10, 11]. In articles [12, 13], as well as in the existing methodology of structural and engineering humping calculations, this problem was solved using the concept of "brake position power." Note that the authors of works [12, 13], when performing humping calculations in both high-velocity and braking areas, do not use formulas to determine railcar [negative] acceleration.

The engineering problem of determining the braking time and distance of a railcar in retarder position sections is poorly studied.

The objectives of this study are building mathematical models of railcar movement based on classical provisions of theoretical mechanics and developing formulas to 
determine braking distance; using the modeling outcomes to confirm the correctness and applicability of the mathematical models for railcar BA in second and third brake positions.

\section{Materials and Methods}

This study offers four solutions to the engineering problem of determining the kinematic characteristics of a railcar based on:

a) the basic law of dynamics for imperfect connections (D'Alembert's Principle)

b) the theorem on the motion of the inertia center of a system of point objects

c) the theorem on change in the kinetic energy of a point object in final form

d) the theorem on the change in the momentum of a point

We write the theorem on change in the kinetic energy of a point object on segment $A B$ [10], where the car can move, factoring in initial velocity $v_{0}$ as applies to the problem under consideration, in the form:

$$
\frac{G}{2 g}\left(v_{B}^{2}-v_{A}^{2}\right)=A_{F x}
$$

Where $v_{A}$ is the velocity at point $A$ (the beginning of motion), $v_{B}$ is the velocity at point $B$ (end of motion), $G$ is gravity, $g$ is gravitational acceleration of $9.8 \mathrm{~m} / \mathrm{s}^{2}$.

$$
A_{F x}=A_{G x}+A_{F f}
$$

Where $A_{G x}$ is a projection of the force of gravity $G x$ on the $x$ axis with the movement of $x_{A B}$ between points $A$ and $B$ ).

$$
{ }^{A_{G x}}=G_{x} x_{A B}=G \sin \psi x_{A B}
$$

$A_{F f}$ is the work of friction force $F_{f}$ (or generally any resistance forces $F_{r}$ ) with the movement of $x_{A B}$ between points $A$ and $B[10]$.

$$
A_{F f}=-F_{f} x_{A B}=-k_{f} G \cos \psi x_{A B}
$$

Where $k_{f}=0.25$ is the coefficient of friction of the wheels of a railway car on rail threads [24], $\psi$ is the angle of inclination of section $A B$ of the hump profile.

The increment $\Delta E$ of the system's kinetic energy is equal to the sum of the corresponding work of active forces $A_{G x}$ and constraint reactions $A_{F f}$ :

$$
\Delta E=A_{G x}+A_{F f}
$$

Substituting (3) and (4) into (2), taking into account (1), after simplifications, we can obtain a formula for determining the velocity of a car in a BA in a retarder position:

$$
v_{B i}^{2}-v_{A i}^{2}=2 g\left(\sin \psi_{i}-k_{f} \cos \psi_{i}\right) x_{A B i}
$$

Where $i$ refers to the numbers of path profile sections $(i=1, \ldots 9), v_{A i}$ is the velocity at point $A_{i}$ (the beginning of movement at the $i$-th section of the profile), $v_{B i}$ is the velocity at 
point $B_{i}$ (the end of movement at the $i$-th section of the profile), $\psi_{i}$ is the angle of incline of section $A i B i$ of the hump profile; or when $x_{A B i}=l_{i}$ the braking distance on the $i$-th section of the profile is

$$
v_{B i}^{2}=v_{A i}^{2}+2 g\left(\sin \psi_{i}-k_{f} \cos \psi_{i}\right) l_{i}
$$

Hence, when $v_{B i}=0$

$$
0=v_{A i}^{2}+2 g\left(\sin \psi_{i}-k_{f} \cos \psi_{i}\right) l_{i}
$$

From formula (7) we obtain braking distance $l_{i}$

$$
l_{i}=\frac{v_{A i}^{2}}{2 g\left(k_{f} \cos \psi_{i}-\sin \psi_{i}\right)}
$$

If we consider that for small angles (less than $5^{\circ}$ ) with the profile along the entire length of the hump yard path $\sin \psi_{i} \approx \psi_{i}=i_{i}$ and $\cos \psi_{i} \approx 1$, then formulas (6) and (8) will look like:

$$
\begin{gathered}
v_{B i}^{2}=v_{A i}^{2}+2 g\left(i_{i}-k_{f}\right) l_{i} \\
l_{i}=\frac{v_{A i}^{2}}{2 g\left(k_{f}-i_{i}\right)}
\end{gathered}
$$

As we see, the braking distance $l_{i}$ is directly proportional to the square of the initial velocity $v_{A i}$ and inversely proportional to friction coefficient $k_{f}$ and the slope of path $i_{i}$.

The absolute value of car [negative] acceleration $\left(\left|a_{i}\right|\right)$ with equally slowed-down motion in the BA is found using the formula [10]:

$$
\left|a_{i}\right|=\frac{\left|\Delta F_{f i}\right|}{M_{r 0}} 10^{3}
$$

Where $\left|\Delta F_{f i}\right|$ is the resulting force under the influence of which the car's wheel pairs are forced to slide along the rolling surfaces of the rail threads and the brake buffers of the car retarder in BA in RP sections [10], defined as

$$
\left|\Delta F_{f i}\right|=F_{x i}+\left|F_{c i}\right|
$$

Where $F_{x i}$ is the force that moves the car into the BA in RP sections, taking into account the influence of a small magnitude tailwind force, $F_{\mathrm{c} i}$ in general refers to all kinds of resistance (resistance to dry sliding friction of the contact surfaces of the wheelset rims and the brake buffers of the car retarder, primary (or running) resistance, resistance from air and wind, snow and hoarfrost resistance) under the influence of which the car can be braked until completely stopped by the car retarder. $M_{r 0}$ is the resulting mass of the wagon with its load and non-rotating parts (i.e. the wagon body and railroad trucks) when the wheelset, 
forced by "compressed" brake buffers in the car retarder in BA in RP sections, slides cleanly like the dry friction pair "steel on steel."

If we know the [negative] acceleration value $\left|a_{i}\right|$ from formula (12) with equally slowed-down car movement, then we can determine the velocity until the car stops using the velocity formula:

$$
v_{f i}^{2}=v_{A i}^{2}+2\left|a_{i}\right| l_{i}
$$

Using the velocity formula

$$
t_{i}=\frac{v_{A i}-v_{f i}}{\left|a_{i}\right|}
$$

we can find the braking time $t_{i}$ until the car stops $t_{i}<t$ - where $t$ is the current time in seconds

$$
t_{i}=\frac{v_{A i}-v_{f i}}{\left|a_{i}\right|}
$$

Thus, applying the theorem on change in the kinetic energy of a point object in its final form in railcar braking areas in RP sections using formulas (8) or (10) made it possible to determine the railcar's distance in braking section $l_{i}$.

To calculate $l_{i}$, the following options are considered:

a) the direct entry of the first wheelset $l_{A i}$ and/or the wheel pairs of the front truck $l_{s t}$ into the retarder position section

b) the entrance of the car to the site based on the car base's length

which are necessary to establish the initial velocity of the car's entry into the braking area $v_{A i}=3.57 \mathrm{~m} / \mathrm{s}$.

The car's braking distance can be determined using the formula

$$
l_{i}=v_{A i} t_{i}+\frac{1}{2} g\left(\sin \psi_{i}-k_{f} \cos \psi_{i}\right) t_{i}^{2}
$$

The car's [negative] acceleration during equally slowed-down movement in the braking area $\left|a_{i}\right|$, unlike formula (12), can be defined as [10].

$$
\left|a_{i}\right|=a_{f}\left(i_{f i}-\left|w_{i}\right|\right)
$$

Where $a_{f}=$ const is the linear [negative] acceleration of the car during equally sloweddown movement in braking areas in RP sections [10], defined as:

$$
a_{f}=\frac{G}{M_{r 0}} 10^{3}
$$

$i_{f i}$ is a dimensionless quantity describing the slope of the hump profile in RP sections when taking into account the projected influence of tailwind force $F_{w x}$ [15-18], which is defined as 


$$
i_{f i}=i_{f x i}+k_{w x}
$$

Where $k_{w x}$ is a dimensionless quantity that considers the projected influence of small magnitude tailwind force $F_{w x}$ on the $x$ axis, contributing to the accelerated movement of the car in fractions of $G . k_{w x}=0$ in the absence of wind. $\left|w_{i}\right|$ is the specific resistance to movement in braking areas in RP sections [10].

Now (16), following (17), can be written as:

$$
l_{i}=v_{A i} t_{i}+\frac{1}{2} a_{f}\left(i_{f i}-\left|w_{i}\right|\right) t_{i}^{2}
$$

and taking into account (17) and (20), it can be represented as

$$
l_{i}=v_{A i} t_{i}+\frac{1}{2} a_{f}\left|a_{i}\right| t_{i}^{2}
$$

Railcar stop time $t_{i}$ is defined as

$$
t_{i}=\frac{v_{A i}}{g\left(k_{f} \cos \psi_{i}-\sin \psi_{i}\right)}
$$

After comparing formula (16), obtained according to the theorem on the motion of the inertia center of a system of point objects, and formula (8), derived using the theorem on change in the kinetic energy of a point object in its final form, with the formula of the distance taken from elementary physics (21), they are clearly different in form.

The relative error in calculating the car braking distance using formulas (21) and (16) was $1.52 \%$, and with formulas (21) and (20) it was $9.2 \%$, which is within a reasonable window of accuracy for engineering calculations.

The car braking distance calculated by the formula $(21)$ is $l_{i}=13.35 \approx 13.4 \mathrm{~m}$, and with formula (9) $l_{i}=12.86 \approx 12.9 \mathrm{~m}$. The relative calculation error is $3.7 \%$, which confirms the correctness of formula (8) outcomes.

\section{Results and Discussion}

To analyze the results, graphic representations of braking distance versus braking time were constructed based on formulas (21), (16) and (20) with $t$ varying from 1.0 to 2.0 with a step of $\Delta t=0.1 \mathrm{~s}$ (Figure 1). 


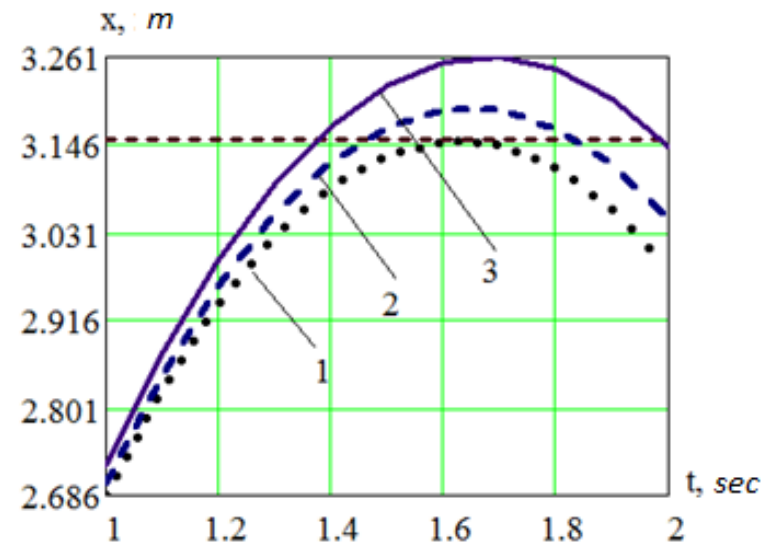

Fig. 1. The dependencies of braking distance on braking time: 1 is according to formula (21); 2 is according to formula (16); 3 is according to formula (20)

The figure 1 graphs show an increasing quadratic function until the car stops. The maximum values for braking distance $l_{i}=3.152,3.195$ and $3.262 \mathrm{~m}$ correspond to braking times of $t_{i}=1.625,1.648$ and $1.682 \mathrm{~s}$.

The graphical dependence of braking distance on velocity, constructed using formula (8) with a variation of $v_{A i}$ from 0 to $5 \mathrm{~m} / \mathrm{s}$ with the step $\Delta v_{A i}=0.25 \mathrm{~m} / \mathrm{s}$, is shown in Fig. 2.

$$
\text { l, } m
$$

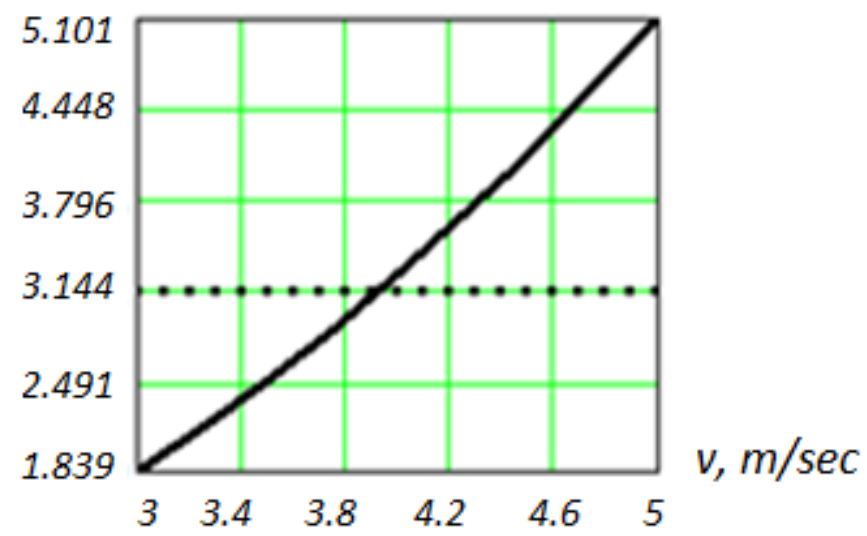

Fig. 2. Dependence of braking distance on velocity

Figure 2 shows how at $v_{A i}=0$, the braking distance is $l_{i}=0$. This confirms the argument about the importance of a railcar entering the braking area at retarder positions with an initial velocity of $v_{A}>0$; otherwise, the car stops completely before the car retarder is engaged.

When $v_{A}>0$, the kinetic energy $E_{0}$ of the car with mass $M$ and initial velocity $v_{A}$ will be completely expended to overcome the work $A_{r}$ and drag force $F_{r}$ which occur when the car retarder is engaged.

In turn, the work of various resistance forces $A_{r}$ accumulated on the rim of the car's wheelsets, on rail threads and on the brake buffers of the car retarder will dissipate into the environment in the form of heat. When the car is completely stopped, i.e. $v_{B}=0$, the following condition will be met: $E_{0}+\left(-A_{r}\right)=0$. 
In [11], it was noted that the existing methodology for humping calculations in hump yards $[13,21,22,23]$ is mainly aimed at determining the height of the hump yard from its top to the calculated point. Such kinematic parameters of railcar movement as [negative] acceleration and movement time in the braking area are not considered. The car's braking distance is also not calculated.

Calculating a railcar's braking distance using formula (8) based on an elementary physics velocity formula (10) made it possible to observe that using the same initial velocity value gives the same results.

In summarizing the results of our calculations of railcar braking time and distance using formulas (8), (10) and (16), (20), (21) - (23), we can note that using the same initial velocity values yielded acceptable results for engineering calculations. This confirms the correctness of our mathematical models as applied to a railcar BA in all retarder position sections (RP1 and RP3).

Mathematical models for the movement of a railcar (chain) along the entire length of an RP section of a hump yard under the influence of a small magnitude tailwind made it possible to develop a new methodology for calculating railcar dynamics in this yard section, which allows the kinematic parameters of the car (rate and velocity) for a given geometric parameter (length) to be determined for the section of the yard under consideration.

Calculations to determine the kinematic parameters of a car according to the new methodology made it possible to determine, for a known travel distance along the entire length of the RP section ("per pass"), the time required for the uniform acceleration of a car in this section of the hump.

\section{Conclusions}

The task of a railway station is to sort railcars from incoming trains and build outgoing trains by appropriately grouping specific classifications of cars. The classification process involves the physical movement of trains, cars and engines between receiving tracks, hump yards and departure tracks. When designing a hump yard profile and the corresponding retarder system, the hump should be high enough to provide railcars with enough kinetic energy to easily overcome rolling resistance and track resistance and roll an estimated distance beyond the touch point.

When the car reaches the beginning of the track (the touch point), it may need to roll back a distance of 30 to $1000 \mathrm{~m}$, depending on the number of cars already standing on the track it is switching to. Due to the large difference in distances and the behavior of cars during rolling, the velocity of each car should be regulated so that no serious collisions occur. At the same time, the cars must have enough energy to connect with other cars that are already waiting on classification routes. The results of this study can be used to adequately address problems in calculating and designing hump yards.

\section{References}

1. O. Kondo, Y. Yamazaki Simulation Technology for Railway Vehicle Dynamics // Nippon Steel \& Sumitomo Metal Technical Report (105). December 2013. pp. 77-83. (2013).

2. V.YA. Negrej, S.A. Pozhidaev, E.A. Filatov Obosnovanie urovnya tekhnicheskogo osnashcheniya i optimizaciya parametrov konstrukcii sortirovochnyh kompleksov zheleznodorozhnyh stancij, Transportni sistemi ta tekhnologiï perevezen'. Zbirnik naukovih prac' DNUZT. im. Akad. V. Lazaryana, pp. 110-119. (2014). 
3. Bardossy M. Analysis of Hump Operation at a Railroad Classification Yard, In Proceedings of the 5th International Conference on Simulation and Modeling Methodologies, Technologies and Applications (SIMULTECH-2015) pp. 493-500. DOI: 10.5220/0005546704930500. (2015).

4. Nils Boysen, Simon Emde, Malte Fliedner The basic train makeup problem in shunting yards // OR Spectrum January 2016, (38), 1, pp 207-233. (2016).

5. Chenxu Lu, Jin Shi, Dynamic response of vehicle and track in long downhill section of high-velocity railway under braking condition, Advances in Structural Engineering. 2019. doi.org/10.1177/1369433219870573 (2019).

6. Dick, C. T., Dirnberger, J. R. Advancing the science of yard design and operations with the CSX hump yard simulation system. Proceedings of the 2014 Joint Rail Conference, pp 1-10. (2014).

7. S.O. Bantyukova Trains Breaking-up Safety Control at Hump Yards, EasternEuropean Journal of Enterprise Technologies. 3, (75) p. 3 (2015)

8. Organization for Co-Operation between Railways (OSJD), Operational and Technical Requirements for the Hump Yards. p 840. Warsaw, (2018).

9. D.M. Kozschenko, V.I. Bobrovsky, S.V. Grevtsov, M.I. Berezobyi. Controlling the Velocity of Rolling Cuts in Conditions of Reduction of Brake Opwer of Car Retardes. Nauka ta progress transportu. Visnik Dnipropetrovs'kogo nacion. univer. zaliznichnogo transportu, 2016.3 (63). - S.28-40. ISSN 2307-3489. (2016).

10. Turanov Kh.T. O matematicheskom opisanii tormozheniya vagona na sortirovochnoj gorke / Kh.T. Turanov, A.A. Gordienko, Sh.U. Saidivaliev // Transport: nauka, tekhnika, upravlenie. 2019, (7). pp. 27 - 30. ISSN 0236-1914. (2019)

11. Turanov Kh.T. O podhode $\mathrm{k}$ opredeleniyu nekotoryh kinematicheskih parametrov dvizheniya vagona na tormoznyh poziciyah sortirovochnyh gorok, Kh.T. Turanov, A.A. Gordienko, Sh.U. Saidivaliev // International Journal of Advanced Studies. 2018, (8),4. pp. 122 - 136. DOI: 10.12731/2227-930X-2018-4-122-136. ISSN 0236-1914. (2018).

12. Rudanovskij V.M. O Popytke kritiki teoreticheskih polozhenij dinamiki skatyvaniya vagona po uklonu sortirovochnoj gorki, V.M. Rudanovskij, I.P. Starshov, V.A. Kobzev , Byulleten' transportnoj informacii. 2016. 6 (252). pp. 19-28. ISSN 20728115. (2016).

13. Pozojskij Yu.O. K voprosu dvizheniya vagona po uklonu zheleznodorozhnogo puti, YU.O. Pozojskij, V.A. Kobzev, I.P. Starshov, V.M. Rudanovskij // Byulleten' transportnoj informacii. 2 (272), pp. 35-38. ISSN 2072-8115. (2018)

14. O. Polach, Creep forces in simulations of traction vehicles running on adhesion limit. Wear, 258 (1), pp 992- 1000, (2005)

15. K. Turanov, A. Gordienko, S. Saidivaliev, S. Djabborov. Designing the height of the first profile of the marshalling hump, E3S Web of Conferences, (164), 03038 (2020). https://doi.org/10.1051/e3sconf/202016403038. (2020).

16. Adilkhodjaev A., Hasanov B., Shaumarov S., Kondrashchenko V. Aerated concrete with predetermined pore parameters for the exterior walls of energy efficient buildings, IOP Conference Series: Materials Science and Engineering, 1030(1), 012006, (2021)

17. K. Turanov, A. Gordienko, S. Saidivaliev, S. Djabborov. Movement of the wagon on the marshalling hump under the impact of air environment and tailwind, E3S Web of Conferences, (164), https://doi.org/10.1051/e3sconf/202016403041 (2020)

18. Khasanov B., Vatin N., Mirzaev T., Suyunov A., Radjabov M. Analysis of the mode of squeezing out excess water for mixing concrete mixture in the process of peristaltic compaction, IOP Conference Series: Materials Science and Engineering, 1030(1), 012021, (2021) 
19. Turanov K., Gordienko A., Saidivaliev S., Djabborov S., Djalilov K. (2021) Kinematic Characteristics of the Car Movement from the Top to the Calculation Point of the Marshalling Hump. In: Murgul V., Pukhkal V. (eds) International Scientific Conference Energy Management of Municipal Facilities and Sustainable Energy Technologies EMMFT 2019. Advances in Intelligent Systems and Computing, vol 1258. Springer, Cham. https://doi.org/10.1007/978-3-030-57450-529, (2019).

20. Kattakulov F., Muslimov T., Khusainov A., Vokhidov O., Sultanov S. Water resource saving in irrigation networks through improving the efficiency of reinforced concrete coatings, IOP Conference Series: Materials Science and Engineering, 883(1), 012053, (2020)

21. Rybakov V., Jos V., Raimova I., and Kudryavtsev K. Modal analysis of frameless arches made of thin-walled steel profiles. IOP Conf. Ser. Mater. Sci. Eng. 883, (2020)

22. Khasanov B., Vatin N., Mirzaev T., Suyunov A., Radjabov M. Physicochemical fundamentals of modifying concrete mix and concrete, IOP Conference Series: Materials Science and Engineering, 1030(1), 012022, (2021)

23. K.T. Turanov, S.U. Saidivaliev, D.I. Ilesaliev. Determining the kinematic parameters of railcar motion in hump yard retarder positions, K.T. Turanov, S.U. Saidivaliev, D.I. Ilesaliev, Structural integrity and life vol. (20), no 2, pp. 143-147. (2020) 